\title{
Líneas prohibidas de alta ionización en una muestra de cuásares
}

\author{
Giovanni Cardona ${ }^{1, *}$, José Gregorio Portilla ${ }^{2}$ \\ ${ }^{1}$ Universidad Pedagógica Nacional \\ ${ }^{2}$ Observatorio Astronómico Nacional, Facultad de Ciencias, Sede Bogotá, Universidad Nacional de Colombia
}

\begin{abstract}
Resumen
La identificación simultánea y el estudio de las líneas prohibidas de alta ionización [Fe VII] $\lambda 6087$ y [Ne V] $\lambda 3426$ en cuásares es el interés de este artículo. Para tal efecto, se estudió una muestra de 1423 espectros de cuásares en el rango óptico obtenidos del Sloan Digital Sky Servey (SDSS) con un criterio de selección para el valor de corrimiento al rojo de $0.14 \leq z \leq 0.2$. De estos objetos solo 18 presentaron las líneas de interés de forma simultánea. Con la razón del flujo de estas líneas y otras cercanas se realizó un diagrama de diagnóstico que permitió contrastar con modelos de fotoionización con el fin de determinar condiciones físicas para la zona de emisión de las líneas coronales.
\end{abstract}

Palabras clave. Cuásar, línea coronal, código de fotoionización

\begin{abstract}
The simultaneous identification of the high ionization forbidden lines [Fe VII] $\lambda 6087$ and [Ne V] $\lambda 3426$ in spectra of quasars is the main interest of this paper. In order to obtain information of these lines we examined a sample of 1423 spectra of quasars obtained from the Sloan Digital Sky Servey (SDSS) utilizing a selection criteria for the redshift $(0.14 \leq z \leq 0.2)$. We only detect 18 objects with the two lines of interest. With the fluxes of coronal lines and other forbidden lines we constructed a diagnostic diagram which allowed us to superimpose photoionization models carried out in CLOUDY in order to determine suitable physical conditions of the emission zone.
\end{abstract}

Key words. Quasar, coronal lines, photoionization code

\section{Introducción}

Entre las líneas de emisión identificadas en los espectros de algunas galaxias activas están las líneas coronales (LCs). Se designan con este nombre a líneas de emisiones que surgen de transiciones prohibidas provenientes de especies químicas altamente ionizadas. Ejemplo de ello son [Fe VII] $\lambda 5721 \AA$ y [Fe XIV] $\lambda 5303 \AA$. Las líneas coronales han sido identificadas y estudiadas en galaxias
Seyfert hace más de 60 años y han sido detectadas también en otros objetos astrofísicos de características energéticas notables, como los remanentes de supernovas y nebulosas planetarias. El nombre "coronales" se debe a que tales emisiones fueron descubiertas por primera vez en la corona solar. Desde el punto de vista físico las líneas coronales son aquellas que se originan por transiciones prohibidas de especies químicas con potenciales de ionización del orden o mayor a 100 eV (Oliva, 1997).

*Correspondencia: Giovanni Cardona, gicardona@hotmail.com

Recibido: 5 de mayo de 2015

Aceptado: 9 de julio de 2015 
A pesar de su relativamente poca intensidad, que en muchos casos apenas se distinguen del nivel del ruido, las líneas coronales se detectan en los espectros de algunos cuásares (Appenzeller \& Wagner, 1997). Estos son objetos cuyo espectro se caracteriza por presentar un gran corrimiento al rojo $(z)$ con notables variabilidades en varias zonas del espectro electromagnético. Se suelen interpretar como núcleos de galaxias activas ubicados a enormes distancias. Autores como Véron-Cetty \& Véron (2006) los definen, para distinguirlos de las galaxias Seyfert y los LINERs, como núcleos de galaxias con magnitudes absolutas en el azul $\left(M_{B}\right)$ más brillantes de -22.25 .

En esta comunicación nos hemos concentrado en las líneas coronales que suelen aparecer con más frecuencia en un espectro óptico de un cuásar con moderado corrimiento al rojo: [Fe VII] $\lambda 6087$ y [Ne V] $\lambda 3426$, las cuales, presumiblemente, son generadas en la misma región, pues sus densidades críticas son semejantes $\left(1.6 \times 10^{7} \mathrm{~cm}^{-3}\right.$ y $3.6 \times 10^{7} \mathrm{~cm}^{-3}$ respectivamente) y sus potenciales de ionización son casi idénticos (97 y $99 \mathrm{eV}$, respectivamente). Aquí se investiga la dependencia de la emisión de las líneas coronales con respecto a condiciones físicas de la zona de emisión basados en modelos de fotoionización los cuales permiten encontrar valores pertinentes para la densidad, nivel de ionización y densidad columnal de la zona emisora.

\section{Muestra de cuásares}

El Sloan Digital Sky Survey (SDSS) una prospección del cielo fotométrica y espectroscópica que ha cubierto más de 1/3 parte del cielo, a través de observaciones hechas con un telescopio de $2.5 \mathrm{~m}$ situado en el observatorio Punto Apache, Nuevo México (York et al., 2000). Se estima que, en la actualidad, gracias a tal prospección existen más de 500 millones de objetos con observaciones fotométricas y unos 3 millones de objetos con sus espectros en el óptico, información esta disponible para cualquiera que desee consultarla a través de su página en internet ${ }^{1}$. El método óptimo para obtener información de forma masiva del servidor del SDSS es realizar una consulta SQL que es la sigla en inglés de Lenguaje Estructurado de Peticiones. La consulta ingresa al servidor de la base de datos del SDSS y genera la muestra, la cual se puede modificar según el interés de cada usuario para ser aplicada en cantidades limitadas de tiempo. Es importante señalar que, al momento de la realización de dicha búsqueda, el Data Release disponible en ese entonces era el DR7 (Abazajian et al., 2009).
En lo que concierne al asunto que nos ocupa aquí, la siguiente secuencia de comandos permitió realizar la consulta de nuestro interés:

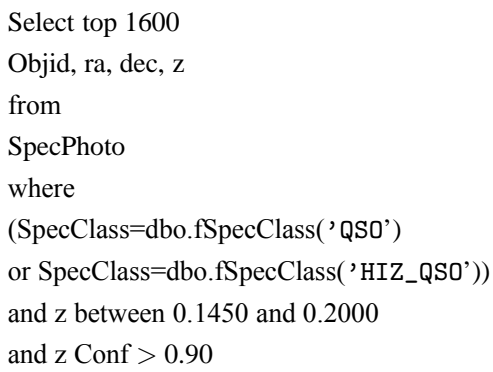

Esta secuencia de comandos arroja un listado de objetos de los 1600 cuásares solicitados con un intervalo especificado de $z$ con cierto nivel de confianza. La consulta arrojó 1423 objetos. Al concentrar nuestro interés en las líneas [Fe VII] $\lambda 6087$ y [Ne V] $\lambda 3426$ a través de una inspección visual de cada espectro, solo 18 objetos (ver Tabla 1) presentaron estas líneas coronales de forma simultánea. Para la confirmación de la existencia de la línea de emisión se utilizó el criterio de que el flujo debe ser mayor o igual a tres veces la desviación estándar del continuo circundante. Es importante resaltar que en todos los 1405 cuásares restantes se identificó $[\mathrm{Ne} \mathrm{V}] \lambda 3426$ para estos valores de $z$, con diferentes intensidades dependiendo de cada objeto.

\begin{tabular}{|l||c|}
\hline Objeto SDSS J & $z$ \\
\hline $014238.47+000514.7$ & 0.1459 \\
$074227.08+465643.0$ & 0.1678 \\
$080549.74+295441.9$ & 0.1612 \\
$083045.40+450235.8$ & 0.1823 \\
$084635.22+465039.1$ & 0.1473 \\
$090022.27+503138.0$ & 0.1476 \\
$094111.13+492419.5$ & 0.1647 \\
$095246.50+095617.0$ & 0.1628 \\
$100121.05+061558.9$ & 0.1621 \\
$103059.09+310256.0$ & 0.1781 \\
$112728.78+634319.7$ & 0.1643 \\
$122450.55+100545.4$ & 0.1679 \\
$123022.17+662154.6$ & 0.1840 \\
$133141.02-015212.4$ & 0.1454 \\
$150719.93+002905.0$ & 0.1822 \\
$152633.81+465712.4$ & 0.1696 \\
$204717.66-054553.4$ & 0.1433 \\
$230216.84+135723.5$ & 0.1654 \\
\hline
\end{tabular}

Tabla 1: Muestra de cuásares con su valor de corrimiento al rojo.

Los 18 espectros obtenidos fueron corregidos y sometidos a varios procesos utilizando tareas estándar

${ }^{1}$ www.sdss.org 
de IRAF $^{2}$ tales como corrección de la escala de flujo, corrección por corrimiento cosmológico, corrección por extinción galáctica y cambio de formato de fits a texto. Sin embargo, los espectros no fueron corregidos por enrrojecimiento intrínseco. Un ejemplo de los mismos puede observarse en la Figura 1, donde se indican varias líneas de emisión y las dos líneas coronales de interés.

Aquellos 18 espectros seleccionados muestran, en general, una contribución dominante por parte de la región de líneas anchas (BLR por sus siglas en inglés) y las líneas coronales se identifican como características débiles y poco conspicuas. Otras líneas coronales que en ocasiones son observadas en núcleos activos más cercanos tales como [Fe X] $\lambda 6374$ y [Fe XI] $\lambda 7892$ no lo fueron aquí, en razón a la tendencia de la primera en mezclarse con [O I] $\lambda 6364$ y estar fuertemente atenuada por el ala ancha de $\mathrm{H} \alpha \mathrm{y}$, en lo que respecta a la segunda, a su no aparición por estar fuera del rango espectral.

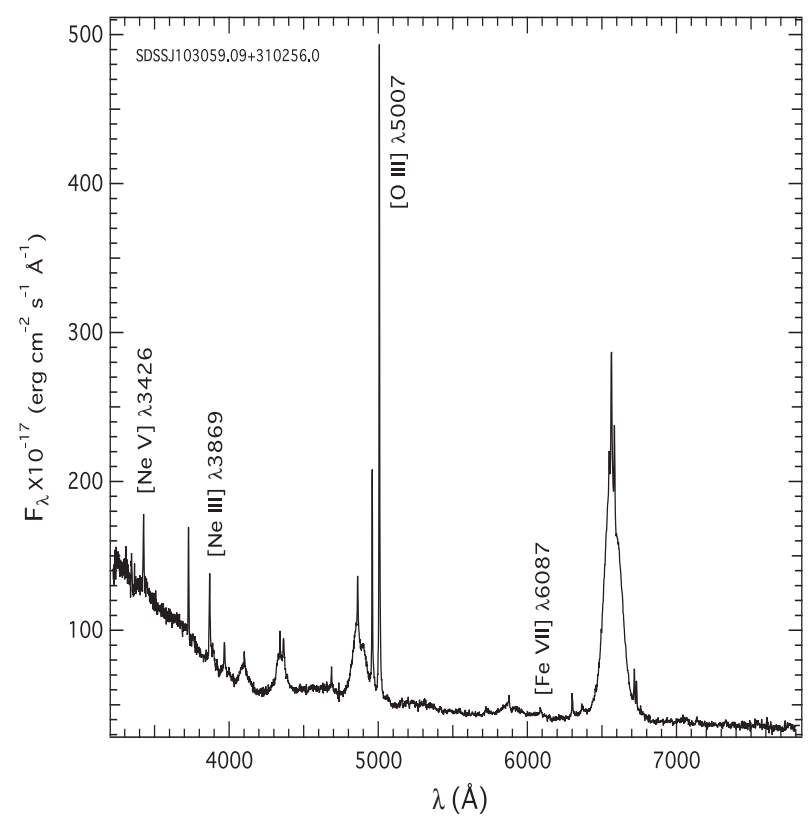

Figura 1: Espectro de emisión en el óptico del cuásar SDSS J103059.09+310256.0.

Se procedió luego a medir el flujo de las líneas de interés pues de estas se puede inferir información sobre las condiciones físicas del gas tales como la densidad y la temperatura electrónica del gas así como también indicios del mecanismo de ionización y excitación (Osterbrock \& Ferland, 2006). De igual forma, del valor del ancho de las líneas a la mitad del valor máximo de flujo, se puede extraer información cinemática del gas. Se asumió que los perfiles de línea pueden ser representados por una o varias gausianas y el continuo debajo de cada línea fue ajustado a un polinomio de orden menor, para lo cual se empleó el programa LINER (Pogge \& Owen, 1993). Se concentraron las observaciones principalmente en las líneas espectrales [Fe VII] $\lambda 6087$ y [Ne V] $\lambda 3426$ y líneas cercanas tales como [Ne III] $\lambda 3869$ y [OIII] $\lambda 5007$ de las cuales se obtuvieron sus respectivos flujos y razones (ver tabla 2).

\begin{tabular}{|l||c|c|}
\hline Objeto SDSS J & $\log ([\mathrm{Ne} \mathrm{V}] /[\mathrm{Ne} \mathrm{III}])$ & $\log ([\mathrm{Fe} \mathrm{VII}] /[\mathrm{O} \mathrm{III}])$ \\
\hline \hline $014238.47+000514.7$ & $0.520 \pm 0.046$ & $-0.802 \pm 0.124$ \\
$074227.08+465643.0$ & $0.144 \pm 0.004$ & $-1.002 \pm 0.088$ \\
$080549.74+295441.9$ & $0.119 \pm 0.012$ & $-1.403 \pm 0.112$ \\
$083045.40+450235.8$ & $-0.021 \pm 0.003$ & $-1.263 \pm 0.063$ \\
$084635.22+465039.1$ & $0.288 \pm 0.039$ & $-1.096 \pm 0.142$ \\
$090022.27+503138.0$ & $0.141 \pm 0.008$ & $-1.064 \pm 0.092$ \\
$094111.13+492419.5$ & $-0.022 \pm 0.046$ & $-1.200 \pm 0.118$ \\
$095246.50+095617.0$ & $-0.089 \pm 0.053$ & $-1.474 \pm 0.152$ \\
$100121.05+061558.9$ & $0.098 \pm 0.026$ & $-1.208 \pm 0.126$ \\
$103059.09+310256.0$ & $-0.189 \pm 0.008$ & $-1.824 \pm 0.127$ \\
$112728.78+634319.7$ & $0.118 \pm 0.042$ & $-0.972 \pm 0.040$ \\
$122450.55+100545.4$ & $0.647 \pm 0.072$ & $-0.803 \pm 0.147$ \\
$123022.17+662154.6$ & $0.082 \pm 0.004$ & $-1.395 \pm 0.100$ \\
$133141.02-015212.4$ & $0.406 \pm 0.060$ & $-1.067 \pm 0.120$ \\
$150719.93+002905.0$ & $-0.228 \pm 0.008$ & $-1.909 \pm 0.115$ \\
$152633.81+465712.4$ & $0.000 \pm 0.019$ & $-1.315 \pm 0.122$ \\
$204717.66-054553.4$ & $0.082 \pm 0.011$ & $-1.421 \pm 0.023$ \\
$230216.84+135723.5$ & $0.133 \pm 0.009$ & $-1.086 \pm 0.105$ \\
\hline
\end{tabular}

Tabla 2: Razones de flujo para las líneas de interés.

Estas medidas permitieron realizar un diagrama donde se contrastó la razón $[\mathrm{NeV}] /[\mathrm{Ne} \mathrm{III}]$ en función de la razón $[\mathrm{FeVII}] /[\mathrm{OIII}]$, ver Figura 2. En la literatura no se han reportado diagramas con estas líneas para cuásares. Los datos observacionales contenidos en este diagrama pueden compararse con resultados de modelos de fotoionización con el fin de determinar las condiciones físicas reinantes en la zona de emisión coronal.

\section{Modelo de fotoionización}

Se ha sugerido que el intenso continuo y las notorias líneas de emisión observadas en los espectros de los núcleos de las galaxias Seyfert y cuásares son, en últimas, debidas a la fotoionización de regiones gaseosas que es originada por una fuente central que emite enormes cantidades de radiación electromagnética (rayos X y UV principalmente). Sin embargo, no es el único: bien puede existir otro mecanismo físico alternativo responsable de la ionización tal como el originado por choques entre nubes que se desplazan a gran velocidad. La principal evidencia para tomar la fotoionización como mecanismo fundamental de generación de las líneas de emisión es

\footnotetext{
${ }^{2}$ IRAF es distribuido por el Observatorio Nacional de Astronomía Óptica que es operado por la Asociación de Universidades para la Investigación en Astronomía (AURA) bajo un acuerdo de cooperación con la Fundación Nacional de Ciencia.
} 
que algunas de estas cambian en respuesta a los cambios en el continuo. La característica fundamental en este caso es que el gas ionizado se encuentra lejos del equilibrio térmico local. El nivel de ionización y de población no están dados por la ecuación de Saha y de Boltzmann, respectivamente, sino por el equilibrio entre los procesos de ionización y recombinación $\mathrm{y}$, en general, de un equilibrio estadístico de todas las poblaciones existentes en el gas (Emerson, 1996; Bowers \& Deeming, 1984). La enorme dificultad en resolver estas ecuaciones se supera con la utilización de poderosos códigos numéricos. Algunos de ellos son de libre acceso y disponibilidad, tales como CLOUDY $^{3}$ (Ferland, 1996) o Mappings (Binette, Dopita \& Tuohy, 1985).

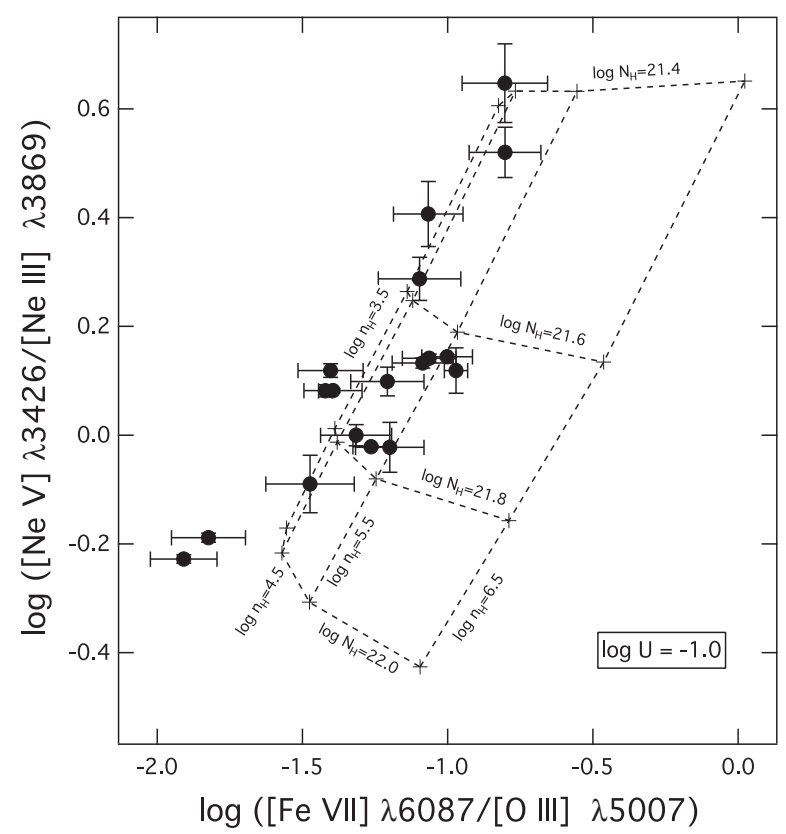

Figura 2: Superposición de los puntos observacionales (puntos negros) con los resultados del modelo de fotoionización con $U=10^{-1}$ variando la densidad entre $10^{3.5}$ y $10^{6.5} \mathrm{~cm}^{-3}$ y la densidad columnal entre $10^{22}$ y $10^{21.4} \mathrm{~cm}^{-2}$.

Con el fin de investigar la región de emisión coronal de la muestra de cuásares desde el marco de la fotoionización se corrieron diferentes modelos usando la versión más reciente de CLOUDY (versión 13.03 (Ferland et al., 2013)). Este código de fotoionización calcula el continuo y los flujos relativos de las líneas de emisión de un gas que es calentado y ionizado por una fuente de radiación central, resolviendo simultáneamente las ecuaciones de equilibrio estadístico y térmico partiendo de un modelo que concibe una nube de geometría esférica fotoionizada

\footnotetext{
${ }^{3}$ www.nublado.org
}

por una fuente central y especificando parámetros de entrada tales como la forma e intensidad del continuo ionizante, la densidad del gas, la densidad columnal y la composición química. Del continuo que ioniza a las nubes debe especificarse tanto su forma como intensidad en un intervalo de energía que está comprendido entre $10^{-8}$ Ryd $(1 \mathrm{Ryd}=13.6 \mathrm{eV})$ a $7.354 \times 10^{6}$ Ryd. A pesar de que se desconoce cómo es la forma funcional del continuo ionizante de un núcleo activo, varias propuestas se han estudiado de dicho perfil para dar cuenta de los espectros estudiados. Usualmente la forma de esos perfiles van desde un cuerpo negro con temperaturas efectivas del orden de $10^{5}-10^{6} \mathrm{~K}$ pasando por combinaciones de leyes de potencia del tipo $f \propto v^{\alpha}$ (siendo $f$ el flujo, $v$ la frecuencia y $\alpha$ un número positivo o negativo) hasta cualesquier composición de estos dos.

Una forma conveniente de escribir la cantidad de energía ionizante generada por la fuente es a través del concepto del parámetro de ionización $U$ :

$$
U=\frac{Q_{H}}{4 \pi r^{2} n_{H} c},
$$

donde $Q_{H}$ es el número total de fotones con energía mayor que 1 Ryd emitida por la fuente ionizante por unidad de tiempo, $r$ es la distancia de la fuente a la nube a ser fotoionizada, $n_{H}$ es la densidad de átomos de hidrógeno contenidos en la nube por unidad de volumen y $c$ es la velocidad de la luz.

Para el caso que nos ocupa se asumió densidad uniforme de las nubes de gas, con una geometría plano paralela y se ejecutaron modelos variando la densidad, el parámetro de ionización $U$ y la densidad columnal $N_{H}$ asumiendo la composición química solar y el continuo ionizante como combinación de varias leyes de potencia.

\section{Rango de parámetros y modelos de fotoionización}

Se asumió el continuo ionizante propuesto por Nagao et al. (2003) en la forma $f \propto v^{\alpha}$ con $\alpha=2.5$ para $\lambda \geq 10$ $\mu \mathrm{m}, \alpha=-1.5$ entre $10 \mu \mathrm{m}$ y $50 \mathrm{keV}$ у $\alpha=-2$ para $h v \geq$ $50 \mathrm{keV}$, el cual es uno de los más comunes para modelar núcleos activos de galaxias. Se consideró que las líneas coronales bien podrían generarse en nubes con densidad semejante a la existente en una típica región $\mathrm{H}$ II pero no tan alta como para alcanzar densidad crítica $\left(\sim 10^{7} \mathrm{~cm}^{-3}\right)$, por lo que se utilizaron valores comprendidos entre $10^{3.5}$ $\mathrm{cm}^{-3}<n_{H}<10^{6.5} \mathrm{~cm}^{-3}$.

Se corrieron diversos modelos con las siguientes características: el parámetro de ionización $U$ se varió 
entre $10^{-2.5}$ a 10 y densidades columnales comprendidas (para la gran mayoría de los casos, salvo en aquellos en donde se diga explícitamente) entre $10^{20}$ y $10^{23.4} \mathrm{~cm}^{-2}$. Con estas variaciones se realizaron varios scripts de entrada como el siguiente:

Interpolate (0.0 -3.388)

(0.0091 4.0115)(3676 -4.398)(10000000 -11.268)

hden 4.5

ionization parameter -1.5

punch continuum "cont.out"

stop effective column density 21.6

print line faint -5

print last

iterate

Las unidades que se utilizan en CLOUDY están en CGS y se pueden introducir de forma logarítmica. Con los resultados de estos modelos se calculó la relación de los flujos de las líneas emergentes de interés [Fe VII] $\lambda 6087$, [Ne V] $\lambda 3426$, [Ne III] $\lambda 3869$ y [OIII] $\lambda 5007$, los cuales generaron una serie de puntos (representados por cruces) para ser contrastados con el diagrama de diagnóstico que contiene los puntos observacionales.

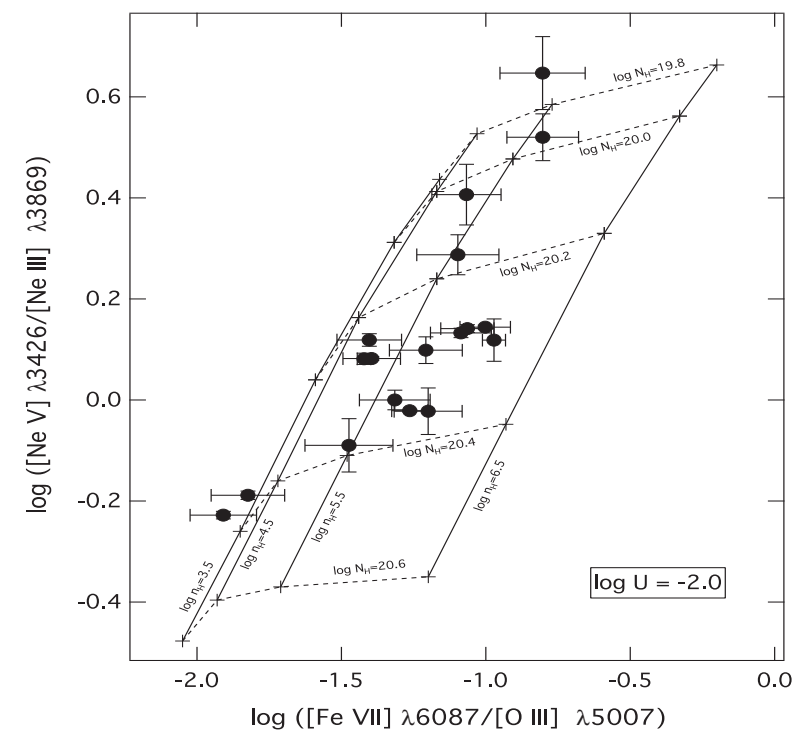

Figura 3: Superposición de los puntos observacionales (puntos negros) con los resultados del modelo de fotoionización con $\mathrm{U}=10^{-2}$ variando la densidad entre $10^{3.5}$ y $10^{6.5} \mathrm{~cm}^{-3}$ y la densidad columnal entre $10^{19.8}$ y $10^{20.6} \mathrm{~cm}^{-2}$.

Las Figuras 2 y 3 presentan el contraste entre los datos obsevacionales (puntos negros) con los resultados del modelo de fotoionización $\left(U=10^{-1}\right.$ y $U=10^{-2}$, respectivamente). Se encontró, para estos dos casos, que la grilla conformada por los puntos del modelo de fotoionización están distribuidas de modo tal que cubren la mayor parte del sector de los puntos observacionales, notándose una mejor sobreposición con $U=10^{-2}$ con rangos de densidad que va de $10^{4.5}$ a $10^{6.5} \mathrm{~cm}^{-3}$. Este comportamiento es coherente con lo reportado por Rodríguez-Ardila et al. (2012), en donde se evidenció, con base en la observación de líneas del $\mathrm{Fe}^{6+}$, que las densidades electrónicas de la zona de emisión coronal tienden a presentarse con mayor frecuencia en el rango de $10^{6}$ a $10^{7} \mathrm{~cm}^{-3}$; adicionalmente, de nuevo para el caso de $U=10^{-2}$, la gran mayoría de los puntos observacionales caen en un rango de densidad columnal entre $10^{20.0}$ y $10^{20.4} \mathrm{~cm}^{-2}$. Al examinar distintos valores del parámetro de ionización superior a $10^{-2}$ es posible encontrar valores de la densidad columnal para los cuales se logra cubrir los puntos observacionales. Sin embargo, en tales casos, el código de fotoionización tiende a cubrir los puntos observacionales con valores de densidad, usualmente comprendidos entre $10^{3.5}$ y $10^{4.5}$ $\mathrm{cm}^{-3}$ y con valores ligeramente crecientes de la densidad columnal aunque en rangos cada vez más estrechos, lo cual no parece ser demasiado plausible. Es el caso presentado en la Figura 4, en el cual se muestran los resultados con un valor del parámetro de ionización de $U=10$.

Por el contrario, al examinar el caso con valores del parámetro de ionización más bajos que $10^{-2}$ se observa que el resultado del código de fotoionización se concentra en zonas bastante alejadas de los puntos observacionales. Como un ejemplo, se muestra en la Figura 5 lo que ocurre cuando se utiliza un parámetro de fotoionización igual a $10^{-2.5}$.

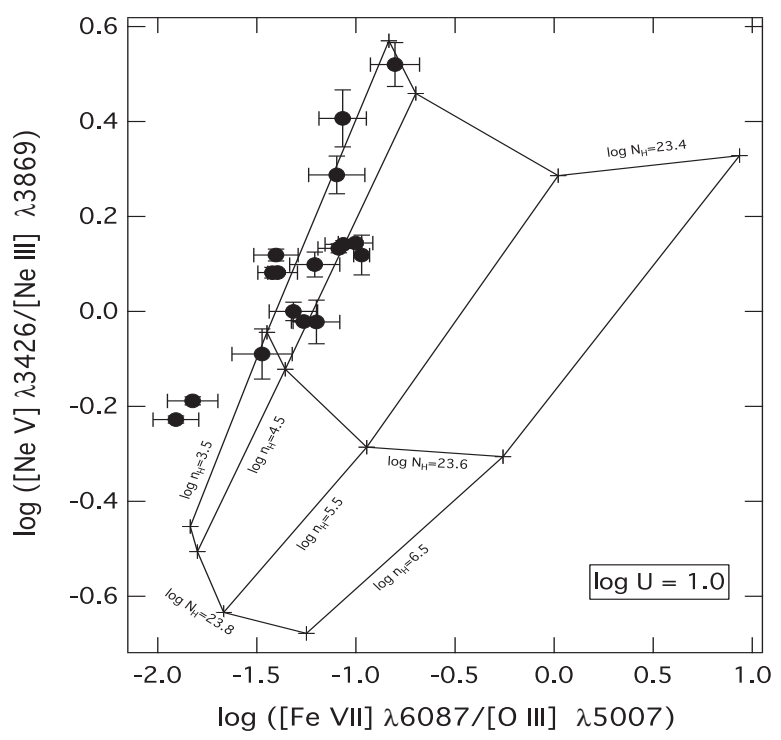

Figura 4: Superposición de los puntos observacionales (puntos negros) con los resultados del modelo de fotoionización con $U=10$ variando la densidad entre $10^{3.5}$ y $10^{6.5} \mathrm{~cm}^{-3}$ y la densidad columnal entre

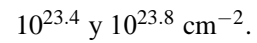




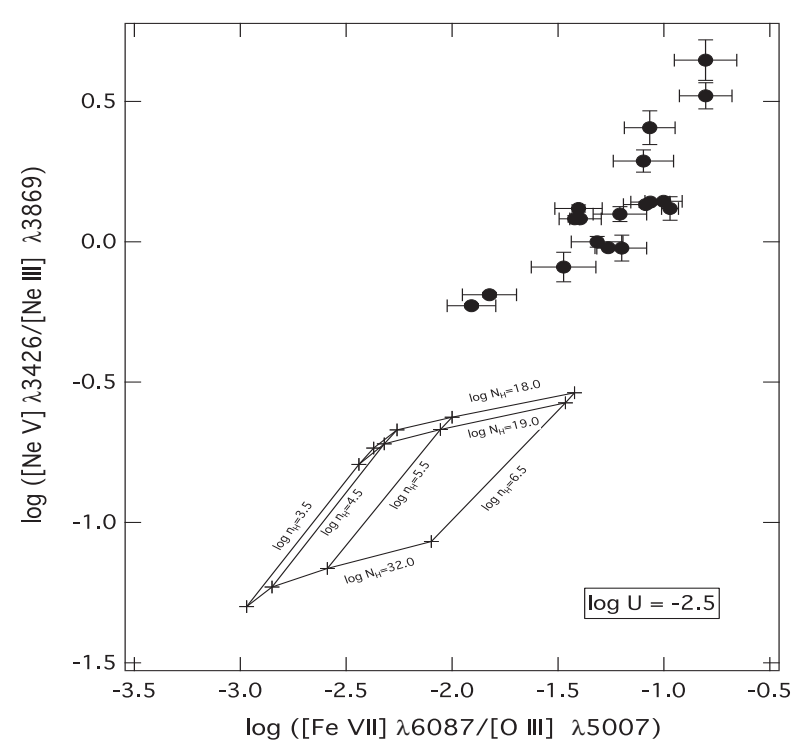

Figura 5: Superposición de los puntos observacionales (puntos negros) con los resultados del modelo de fotoionización con $U=10^{-2.5}$ variando la densidad entre $10^{3.5}$ y $10^{6.5} \mathrm{~cm}^{-3}$ y la densidad columnal

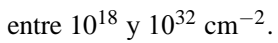

En tal caso solo, el código de fotoionización arroja resultados comprendidos entre valores de densidad columnal anormalmente bajos $\left(N_{H}=10^{18} \mathrm{~cm}^{-2}\right)$ y valores de densidad columnal de $10^{32} \mathrm{~cm}^{-2}$ que es el valor máximo que el código tiene en cuenta. Dichas zonas están bastante alejadas de la zona que corresponde a los puntos observacionales.

\section{Conclusiones}

Contando con el gran número de espectros del SDSS hemos logrado obtener resultados con relación a la emisión coronal existente en objetos clasificados como cuásares.

La línea de $[\mathrm{Ne} \mathrm{V}] \lambda 3426$ fue detectada casi en todos los cuásares examinados en tanto que la línea de [Fe VII] $\lambda 6087$ apareció (con la primera) contundéntemente sólo en 18 objetos. Dos líneas casi con idéntico potencial de ionización y con semejantes densidades críticas deberían aparecer de forma simultánea con más frecuencia en este tipo de espectros. Es posible que esto se deba a un efecto de abundancia química, pues el neón es, al menos para la composición química solar, 10 veces más abundante que el hierro. La deficiencia en la presencia de líneas de hierro también podría indicar el hecho de que este elemento esté atrapado molecularmente conformando de granos que constituyen el polvo presente.
Con esos 18 objetos se construyó un diagrama de diagnóstico lo que permitió, con un modelo en particular usando el código de fotoinización Cloudy, estimar las condiciones físicas de la zona emisora. Se encontró que con un perfil ionizante con un índice $\alpha$ igual a -1.5 en la zona entre el IR y la mayor parte de los rayos X, se genera un rango de valores para una familia de parámetros físicos coherentes que pueden reproducir los valores observados. Éstos se reproducen más adecuadamente para valores del parámetro de ionización entre $10^{-2} \mathrm{y}$ $10^{-1.5}$, con densidades electrónicas comprendidas entre $10^{4.5}$ y $10^{6.5} \mathrm{~cm}^{-3}$. Es importante anotar que esto se logró sin necesidad de recurrir a composiciones químicas anormales. El intervalo de valores de las densidades columnales varía dependiendo de $U$ : a mayores valores de éste, se requieren mayores valores de intervalo de densidad columnal para los cuales se logran reproducir los datos observacionales. Valores superiores del parámetro de ionización arrojan valores de densidad electrónica que ya son relativamente bajos para la zona de emisión coronal; valores más bajos de $U$ que $10^{-2}$ no se acercan a la zona de los puntos observacionales con independencia del valor de la densidad columnal.

El hecho de haber obtenenido un ajuste relativamente exitoso en la explicación de los puntos observacionales utilizando un modelo de fotoinozación como el CLOUDY, con ciertos rangos específicos de valores, permite dar apoyo a la propuesta de que el mecanismo principal de ionización de las especies químicas involucradas sea el fujo intenso de radiación electromagnética (Nussbaumer \& Osterbrock, 1970), con un continuo ionizante que permanece por especificar en su forma y en el que las LCs se producen en zonas con densidades electrónica dos o tres órdenes de magnitud superiores a los de una típica región H II.

\section{Agradecimientos}

Los autores agradecen las sugerencias y las indicaciones de parte de dos árbitros anónimos que permitieron la mejora del artículo.

La financiación para el SDSS y el SDSS-II es suministrada por la fundación Alfred P. Sloan, las Instituciones Participantes, la Fundación Nacional de Ciencia, el Departamento de Energía, la Administración Nacional de la Aeronáutica y del Espacio, la Monbukagakusho del Japón, la Sociedad Max Planck y el Concilio de Financiamiento de Educación Superior para Inglaterra. El sitio de Internet es: http://www.sdss.org/ 


\section{Conficto de interés}

Los autores declaran que no tienen ningún conflicto de interés.

\section{Bibliografía}

Abazajian K., et al., 2009. The Astrophysical Journal Supplement Series, 182, 543.

Appenzeller I., \& Wagner, 1991. Astronomy \& Astrophysics, 250, 57.

Binette L., Dopita M. A., \& Tuohy I. R., 1985. The Astrophysical Journal, 297, 476.

Bowers R. L. \& Deeming T., 1984. Astrophysics II, Jones and Bartlett Pu. Inc., Boston.

Emerson A., 1996. Interpreting Astronomical Spectra, John Wiley and Sons, New York.
Ferland G. J. 1996. Hazy, a brief introduction to Cloudy, Reporte interno de la Universidad de Kentucky.

Ferland G. J., et al. 2013. Revista Mexicana de Astronomía y Astrofísica, 49, 137.

Nagao T., et al. 2003. Astronomical Journal, 125, 1779.

Nussbaumer H., \& Osterbrock D. E., 1970. Astrophysical Journal, 161, 811.

Oliva E., 1997. ASP Conference series, 113, 288.

Osterbrock D., \& Ferland G. J., 2006. Astrophysics of Gaseous Nebulae and Active Galactic Nuclei, University Science Books, Sausalito.

Pogge R. W., \& Owen J. M., 1993. Reporte interno de Ohio State University, 93-01

Rodríguez-Ardila A., et al. 2012. ASP Conference Series, 460, 144.

Véron-Cetty \& Véron 2006. A catalogue of quasars and active nuclei: 12th edition, $\mathbf{4 5 5}, 773$.

York D. G., et al., 2000. Astronomical Journal, 120, 1579. 